\title{
Current-Tuned Superconductor to Insulator Transition in Granular $\mathrm{Sm}_{1.82} \mathrm{Ce}_{0.18} \mathrm{CuO}{ }_{4-\delta}$ Superconductor
}

\author{
M. S. da Luz ${ }^{1}$, M. J. R. Sandim ${ }^{1}$, C. A. M. dos $\operatorname{Santos}^{1}$, A. J. S. Machado ${ }^{1}$, and R. F. Jardim ${ }^{2}$ \\ ${ }^{1}$ Escola de Engenharia de Lorena, USP, P. O. Box 116, 12600-970, Lorena - SP, Brazil \\ ${ }^{2}$ Instituto de Física, USP, C.P. 66318, 05315-970, São Paulo - SP, Brazil
}

Received on 27 June, 2007

\begin{abstract}
We have measured transport properties at low temperatures for the granular $\mathrm{Sm}_{1.82} \mathrm{Ce}_{0.18} \mathrm{CuO}_{4-\delta}$ samples belonging to the family of electron-doped superconductors. The effect of applied electrical current on the resistive behavior is investigated. The experimental data are analyzed using a modified form of the theory for a field-tuned superconductor-insulator transition in 2D superconductors. The results suggest a possible electricalcurrent driven superconductor-insulator transition.
\end{abstract}

Keywords: Superconductor-insulator transition; Granular superconductors; $\mathrm{Sm}_{1.82} \mathrm{Ce}_{0.18} \mathrm{CuO} \mathrm{O}_{4-\delta}$

\section{INTRODUCTION}

The superconductor to insulator transition (SIT) in twodimensional (2D) superconductors has attracted considerable interest in the last years. Such a transition, which can be tuned by the application of external magnetic field, has been object of interest for both theoreticians [1] and experimentalists [2-4]. As far as the theories are concerned an important contribution to the area is particularly related to the occurrence of the SIT in disordered 2D superconducting systems [1]. The theory predicts a finite temperature scaling law by assuming that a disordered 2D superconductor can be defined as either a superconductor or an insulator at $T=0$ provided that $d R / d T>0$ or $d R / d T<0$, respectively, where $R(T)$ is the temperature dependence of the electrical resistance. The scaling function predicted in Ref. 1 for $R(T)$ at moderately magnetic fields and close to the insulator to superconducting transition is given by:

$$
R(H, T)=R_{c r} f\left(\left|H-H_{c r}\right| / T^{1 / z v}\right)
$$

where $R_{c r}$ is the electrical resistance at the transition, $H_{c r}$ is the applied magnetic field at the critical point of the SIT, $\mathrm{f}\left(\left|H-H_{c r}\right| / T^{1 / z v}\right)$ is the scaling function such that $f(0)=1$, and $z$ and $v$ are critical exponents, such that $z v \geq 1$ [1].

Concerning high $T_{c}$ cuprates, the occurrence of the SIT tuned by applying magnetic fields has been reported in singlecrystal and thin films materials [5-7], and very good agreement between experimental data and the scaling function described in Eq. (1) has been obtained [6].

The superconductor to insulator transition is also an important issue within of the scenario of granular superconducting systems. These systems are characterized by two superconducting critical temperatures: $T_{c i}$ and $T_{c J}[8,9]$. The first superconducting transition, $T_{c i}$, which occurs at an upper transition temperature, is attributed to the development of the amplitude of the superconducting order parameter within isolated superconducting grains $[8,9]$. With decreasing temperature, a second transition is observed at $T_{C J}$ and is attributed to the development of Josephson coupling between isolated superconducting islands. Such a transition eventually drives the system to the zero resistance state which is related to the long-range phase ordering of the order parameter through Josephson coupling $[8,9]$.

In these superconducting granular systems the coupling between grains due to the Josephson effect is pronounced and very sensitive to both applied magnetic fields and excitation currents $[8,9]$. Usually, application of low magnetic fields and electrical currents essentially unaffect $T_{c i}$ but results in appreciable changes in the macroscopic properties of the system particularly below $T_{C J}$. Such an effect is usually related to the suppression of the long range order in the phase coherence of the order parameter, where the so called global superconductivity is therefore suppressed [8,9].

Previously, we have reported results suggesting the possibility of a current-induced superconductor-insulator transition in granular high $T_{c}$ superconductors $[10,11]$. The experimental data were analyzed according to a modified form of the scaling law predicted in Ref. 1, in analogy with the field-tuned SI transition. The results presented in Refs. 10 and 11 were concerned to granular samples belonging to the hole-doped system $\mathrm{Y}_{1-x} \mathrm{Pr}_{x} \mathrm{Ba}_{2} \mathrm{Cu}_{3} \mathrm{O}_{7-\delta}(Y \operatorname{Pr} 123)$. On the other hand, the comparison of the transport properties for both electron and hole-doped superconductors is a current issue $[12,13]$. In the present work we focus on the SIT in granular samples of the $\mathrm{Sm}_{1.82} \mathrm{Ce}_{0.18} \mathrm{CuO}_{4-\delta}$ compound. This compound belongs to the family of electron-doped cuprate superconductors $\mathrm{R}_{2-x} \mathrm{Ce}_{x} \mathrm{CuO} \mathrm{O}_{4-\delta}(\mathrm{R}=\mathrm{Nd}, \mathrm{Sm}, \mathrm{Pr})$. The results are compared to the ones obtained for the granular samples belonging to the hole-doped system $Y \operatorname{Pr} 123$ [10,11].

\section{EXPERIMENTAL PROCEDURE}

Polycrystalline samples of $\mathrm{Sm}_{1.82} \mathrm{Ce}_{0.18} \mathrm{CuO}_{4-\delta}$ were prepared by using the sol-gel route [14]. X-ray powder diffraction analysis revealed single phase materials with the T'structure. Details regarding the sample preparation and the reduction process necessary to induce superconducting properties in this compound are given in Ref. 15 .

The transport measurements were performed by using the Maglab Exa-Oxford platform, by means of the standard fourprobe technique using low-resistance $(<1 \Omega)$ sputtered $\mathrm{Au}$ 
contacts. The temperature dependence of the electrical resistance, $\mathrm{R}(\mathrm{T})$, was obtained at zero applied magnetic field and in the temperature range $3<T<30 \mathrm{~K}$. The $R(T)$ curves were taken by using several values of the excitation current, $0.2 \leq I \leq 2 \mathrm{~mA}$. The voltage versus excitation current (I-V) curves were carried out at zero magnetic field for several values of temperature between 2.5 and $5 \mathrm{~K}$. The characterization of transport properties was complemented performing the resistance versus magnetic field curve, $R(H)$, at $T=5 \mathrm{~K}$ for applied magnetic field from zero up to $0.1 \mathrm{~T}$.

\section{RESULTS AND DISCUSSION}

Let us start the discussion by showing in Fig. 1(a) the temperature dependence of the electrical resistance, $R(T)$, for the granular $\mathrm{Sm}_{1.82} \mathrm{Ce}_{0.18} \mathrm{CuO} \mathrm{O}_{4-\delta}$ superconductor. The occurrence of the double resistive superconducting transition is clearly seen in the $R(T)$ data. A careful inspection of the curve also indicates that the upper superconducting transition occurs at $T_{c i} \approx 21 \mathrm{~K}$ and that the lower one at $T_{c J} \approx 10 \mathrm{~K}[8,15]$. An important feature of the $R(T)$ curve concerns the separation between $T_{c i}$ and $T_{c J}$ in temperature. Such a feature, $T_{c J}$ far apart from $T_{c i}$, corresponds to $\sim 11 \mathrm{~K}$, is unusual in superconducting cuprates, and provides an opportunity to investigate separately both inter and intragranular components for the transport properties. In fact, measurements in the temperature range below $T_{C J}$ are particularly useful for studies involving the behavior of the phase of the superconducting order parameter.

The above statement is supported by the results showed in Fig. 1(b), where a hysteresis loop of the electrical resistance under magnetic field $R(H)$ is displayed. Such a hysteresis loop was measured at $T=5 \mathrm{~K}$ and for excitation current of $I=0.2 \mathrm{~mA}$. It is important to notice here that the electrical resistance, corresponding to the increasing branch of $R(H)$, is larger than that corresponding to the decreasing branch, resulting in a clockwise $R(H)$ hysteresis loop. According to Ref. 11 such a feature is compelling evidence that, below $T_{C J}$, the electrical resistance of the granular $\mathrm{Sm}_{1.82} \mathrm{Ce}_{0.18} \mathrm{CuO} \mathrm{O}_{4-\delta}$ superconductor is mainly governed by the motion of Josephson vortices.

A deeper insight onto dissipation mechanisms behind in the investigated compound can be obtained from the data displayed in Fig. 2. This figure shows the temperature dependence of the electrical resistance for the $\mathrm{Sm}_{1.82} \mathrm{Ce}_{0.18} \mathrm{CuO} \mathrm{O}_{4-\delta}$ compound for several values of excitation current in the range $0.2 \mathrm{~mA} \leq I \leq 2 \mathrm{~mA}$. The relevant feature of this figure is a well defined change in the behavior of $d R / d T$ from positive (superconducting state) to negative (insulating state) as $I$ is evolved. We have also found that such a crossover feature in the behavior of $d R / d T$ occurs for a crossover current $I_{c r} \sim 1.5$ $\mathrm{mA}$.

The change in the $d R / d T$ behavior is reflected in the current-voltage I-V characteristics for the investigated compound. Typical I-V isotherms, taken at several temperatures between 2.5 and $5 \mathrm{~K}$, are shown in Fig. 3. The results also indicate a crossing of the $\mathrm{I}-\mathrm{V}$ isotherms close to $I \sim 1.6 \mathrm{~mA}$,
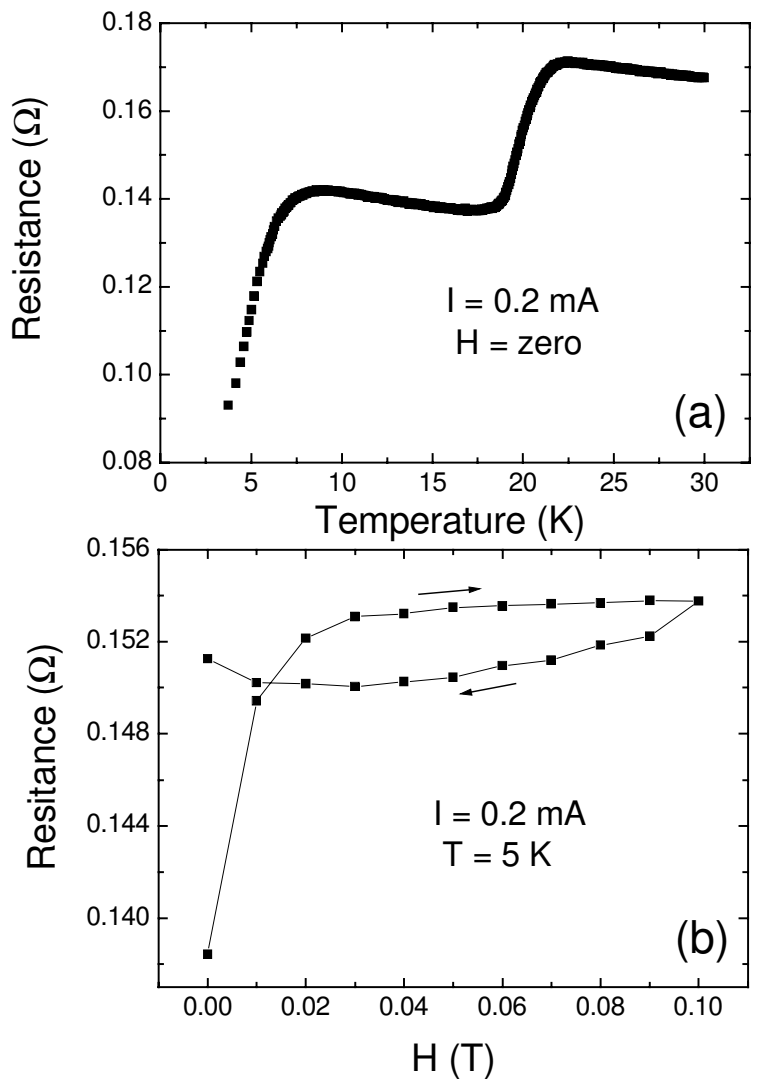

FIG. 1: (a) Temperature dependence of the electrical resistance for the granular $\mathrm{Sm}_{1.82} \mathrm{Ce}_{0.18} \mathrm{CuO}_{4-\delta}$ superconductor measured at an excitation current $I=0.2 \mathrm{~mA}$; (b) Magnetoresistance hysteresis loop measured at $T=5 \mathrm{~K}$. The excitation current used here was $I=0.2$ $\mathrm{mA}$.

a value very close to $I_{c r}$, determined via temperature dependence of the electrical resistance under different $I$ (see Fig. 2). The crossover behavior observed in $R(T)$ curves, displayed in Fig. 2 is similar to the ones predicted in a magnetic field tuned superconductor-insulator transition [1]. Moreover, these results resemble the ones reported for the granular $Y \operatorname{Pr} 123$ superconductor $[10,11]$, which suggested a SIT driven by electric current.

Based on these findings, the occurrence of a similar SIT in the granular $\mathrm{Sm}_{1.82} \mathrm{Ce}_{0.18} \mathrm{CuO} \mathrm{O}_{4-\delta}$ compound was further investigated. Thus, the I-V isotherms displayed in Fig. 3 were analyzed according to Eq. (1), where the parameter B has been replaced by the electric current. By doing this, it is supposed that the role of the excitation current in the dynamics of Josephson vortices in granular superconductors is similar to the one of the magnetic field in the dynamics of Abrikosov vortices in two-dimensional superconductors. Within this context, the electrical resistance in the critical regime of the SIT can be written as:

$$
R=R_{c r} f\left(\left|I-I_{c r}\right| / T^{1 / z v}\right)
$$

where $R=V / I . \quad R_{c r}$ is the resistance at the transition, 


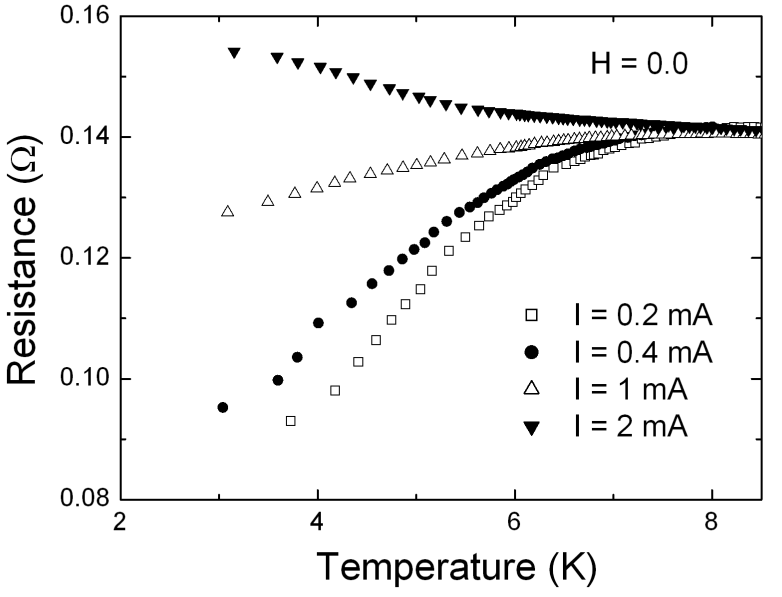

FIG. 2: Temperature dependence of the electrical resistance for the $\mathrm{Sm}_{1.82} \mathrm{Ce}_{0.18} \mathrm{CuO}_{4-\delta}$ compound for several values of the excitation current in the range $0.2 \mathrm{~mA} \leq I \leq 2 \mathrm{~mA}$.

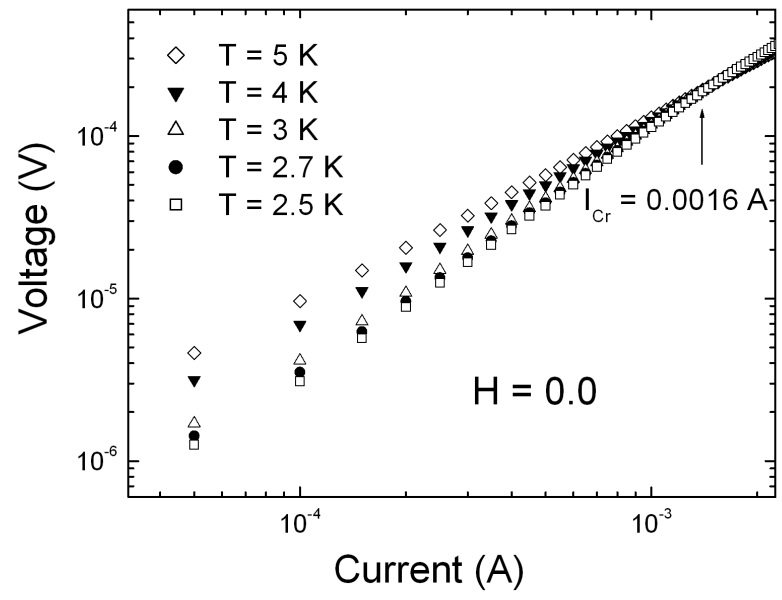

FIG. 3: Current-voltage characteristics taken at several temperatures in the range $2.5 \leq T \leq 5 \mathrm{~K}$. The crossover point $I=I_{c r}$ in the I-V curves is marked with an arrow.

$f\left(\left|I-I_{c r}\right| / T^{1 / z v}\right)$ is the scaling function such that $f(I=$ $\left.I_{c r}\right)=1, I_{c r}$ is the crossover excitation current, $z$ and $v$ are critical exponents.

According to Reference 11 and 16, the Eq. (2) can be rearranged, yielding

$$
|d(V / I) / d I|_{I \sim I_{c r}} \alpha T^{-1 / z v}
$$

or

$$
\ln \left[|d(V / I) / d I|_{I \sim I_{c r}}\right]=-(1 / z \mathrm{~V}) \ln T
$$

Therefore, according to Eq.(4), the critical exponent $1 / z \mathrm{~V}$ can be obtained from the linear behavior of $\ln \left[d(V / I) /\left.d I\right|_{I \sim I_{c r}}\right.$ ] vs. $\ln T$ plots, similar to the one shown in Fig. 4. ¿From the linear fit, one obtains $z \mathrm{v}=0.86$ for the granular superconducting compound $\mathrm{Sm}_{1.82} \mathrm{Ce}_{0.18} \mathrm{CuO} \mathrm{O}_{4-\delta}$ at zero applied magnetic

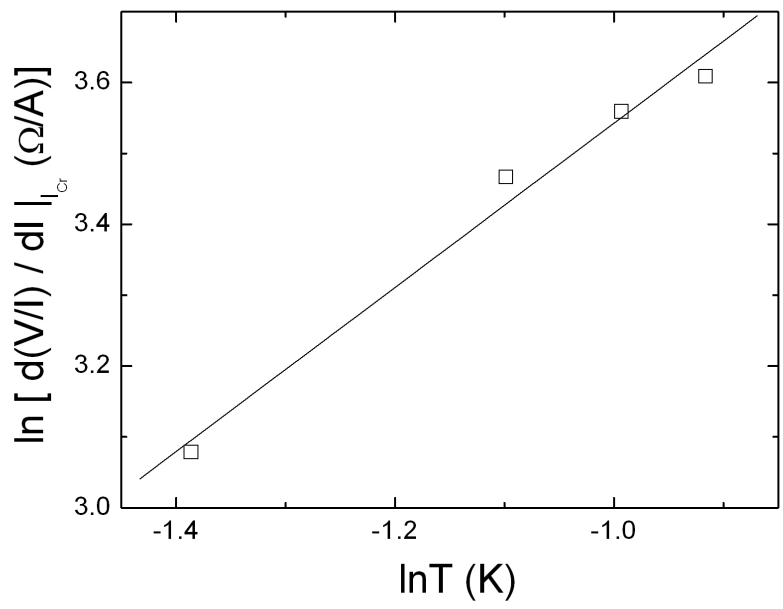

FIG. 4: $\ln \left[d(V / I) / d I_{\left|I \sim I_{c r}\right|}\right]$ as a function of $\ln (T)$ for the granular superconducting $\mathrm{Sm}_{1.82} \mathrm{Ce}_{0.18} \mathrm{CuO}_{4-\delta}$ compound. The value of the critical exponent $z v$ (see Eq. (4)) was obtained from the linear fit of this curve.

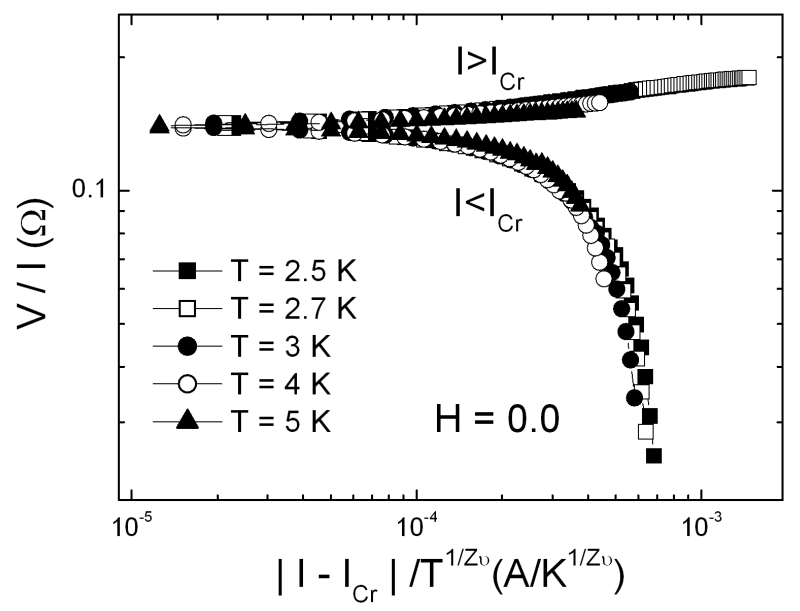

FIG. 5: Electrical resistance $(R=V / I)$ as a function of the scaling variable $\left(\left|I-I_{c r}\right| / T^{1 / z v}\right)$ for the $\mathrm{Sm}_{1.82} \mathrm{Ce}_{0.18} \mathrm{CuO} \mathrm{O}_{4-\delta}$ granular superconductor at zero applied magnetic field. The collapse of the electrical resistance data onto two distinct branches, corresponding to $I<I_{c r}$ and $I>I_{c r}$, respectively, is easily observed from the figure.

field. By using the obtained value of $z \mathrm{~V}$ in Eq. (2), the V/I versus the scaling parameter $\left.\left|I-I_{c r}\right| / T^{1 / z v}\right)$ curves are displayed in Fig. 5. A clear collapse of the electrical resistance values onto the two branches, distinguishing the $I<I_{c r}$ from $I>I_{c r}$ data, is observed.

An important issue in the present analysis is concerned to the obtained $z \mathrm{~V}$ value, at light of the Ref. 1. According to this reference, the predicted value for this critical exponent is such that $z \mathrm{~V} \geq 1$. Indeed, the $z \mathrm{~V}$ value obtained for the granular samples of the system $Y \operatorname{Pr} 123$ are in accordance with the predictions of the Ref. $1[10,11]$. As described above, for the granular superconducting compound $\mathrm{Sm}_{1.82} \mathrm{Ce}_{0.18} \mathrm{CuO} \mathrm{O}_{4-\delta}$, the obtained value for this critical exponent is 0.86. However, it must be noticed that 
the scaling result displayed in Fig. 5 is empiric, as well as the correspondent ones for granular $Y \operatorname{Pr} 123$ superconductors $[10,11]$. In spite of this, the very good scaling fitting observed in Fig. 5, supported by the results of references 10 and 11 , suggests the occurrence of an excitation current induced superconductor-insulator transition in the granular superconducting $\mathrm{Sm}_{1.82} \mathrm{Ce}_{0.18} \mathrm{CuO}_{4-\delta}$ compound.

\section{CONCLUSIONS}

Based on the transport properties measurements for the granular $\mathrm{Sm}_{1.82} \mathrm{Ce}_{0.18} \mathrm{CuO} \mathrm{O}_{4-\delta}$ compound, the following conclusions can be drawn:

a) As $I$ evolves a crossover behavior in the I-V isotherms occurs for a specific current value $I_{c r} \sim 1.6 \mathrm{~mA}$. The $d R / d T$ curve also exhibits a crossover behavior at $I \sim I_{c r}$;

b) The electrical resistance data as a function of the scal- ing variable $\left(\left|I-I_{c r}\right| / T^{1 / z v}\right)$ collapses onto two branches, distinguishing the $I<I_{c r}$ from the $I>I_{c r}$ data. Such a scaling behavior suggests the occurrence of an electricalcurrent-driven superconductor to insulator transition in granular $\mathrm{Sm}_{1.82} \mathrm{Ce}_{0.18} \mathrm{CuO} \mathrm{O}_{4-\delta}$ superconductor. Furthermore, the results further indicate that the current-induced SIT can be considered as the dynamical counterpart of the magnetic-field tuned transition;

c) The results are similar to the ones obtained for the $Y \operatorname{Pr} 123$ system, showing a common feature in the SIT for both hole and electron-doped granular superconductors.

\section{Acknowledgements}

The authors are grateful to the Brazilian agencies FAPESP (Grant No. 97/11113-6, and 00/03610-4), CNPq and CAPES. R. F. J. is a CNPq fellow under Grant No. 303272/2004-0.
[1] M. P. A. Fisher, Phys. Rev. Lett. 65, 923 (1990).

[2] A. F. Hebard, M. A. Paalanen, Phys. Rev. Lett. 65, 927 (1990).

[3] A. Yazdani, A. Kapitulnik, Phys. Rev. Lett. 74, 3037 (1995).

[4] N. Markovic, C. Christiansen, and A. M. Goldman, Phys. Rev. Lett. 81, 5217 (1998).

[5] G. T. Seidler, T. F. Rosenbaum, and B. W. Veal, Phys. Rev. B 45, 10162 (1992).

[6] S. Tanda, S. Ohzeki, and T. Nakayama, Phys. Rev. Lett. 69, 530 (1992).

[7] F. Ichikawa, Y. Yamasaki, T. Nishizaki, T. Fukami, T. Aomine, S. Kubo, and M. Suzuki, Solid State Commun. 98, 139 (1996).

[8] M. J. R. Sandim, R. F. Jardim, Physica C 328, 246 (1999).

[9] C. A. M. dos Santos, M. S. da Luz, B. Ferreira, and A. J. S. Machado, Physica C 391, 345 (2003).

[10] C. A. M. dos Santos, Y. Kopelevich, S. Moehlecke, and A. J. S.
Machado, Physica C 341-348, 1047 (2000).

[11] Y. Kopelevich, C. A. M. dos Santos, S. Moehlecke, and A. J. S. Machado, International Book Series: Studies of high temperature superconductors, Vol. 39, Chapter 8, p. 201, ed. A. V. Narlikar (2001).

[12] Y. Tokura, H. Takagi, and S. Uchida, Nature 337, 345 (1989).

[13] S. Y. Li, W. Q. Mo, X. H. Chen, Y. M. Xiong, C. H. Wang, X. G. Luo, and Z. Sun, Phys. Rev. B 65, 224515 (2002).

[14] R. F. Jardim, L. Ben-Dor, and M. B. Maple, J. Alloys and Compounds 199, 105 (1993).

[15] R. F. Jardim, L. Ben-Dor, D. Stroud, and M. B. Maple, Phys. Rev. B 50, 10080 (1994).

[16] A. F. Hebard, Strongly Correlated Electronic Materials, The Los Alamos Symposium, Addison Wesley (1993). 